\title{
micromachines
}

ISSN 2072-666X

www.mdpi.com/journal/micromachines

Article

\section{Optimized SU-8 Processing for Low-Cost Microstructures Fabrication without Cleanroom Facilities}

\author{
Vânia C. Pinto ${ }^{1, *}$, Paulo J. Sousa ${ }^{1}$, Vanessa F. Cardoso ${ }^{1,2}$ and Graça Minas ${ }^{1}$ \\ 1 Centro Algoritmi, University of Minho, Campus de Azurém, 4800-058 Guimarães, Portugal; \\ E-Mails: psousa@dei.uminho.pt (P.J.S.); vanessa@dei.uminho.pt (V.F.C.); \\ gminas@dei.uminho.pt (G.M.) \\ 2 Centro de Física, University of Minho, Campus de Gualtar, 4710-057 Braga, Portugal \\ * Author to whom correspondence should be addressed; E-Mail: vpinto@dei.uminho.pt; \\ Tel.: +351-253-510-190; Fax: +351-253-510-189.
}

Received: 14 July 2014; in revised form: 9 September 2014 / Accepted: 12 September 2014 / Published: 22 September 2014

\begin{abstract}
The study and optimization of epoxy-based negative photoresist (SU-8) microstructures through a low-cost process and without the need for cleanroom facility is presented in this paper. It is demonstrated that the Ultraviolet Rays (UV) exposure equipment, commonly used in the Printed Circuit Board (PCB) industry, can replace the more expensive and less available equipment, as the Mask Aligner that has been used in the last 15 years for SU-8 patterning. Moreover, high transparency masks, printed in a photomask, are used, instead of expensive chromium masks. The fabrication of well-defined SU-8 microstructures with aspect ratios more than 20 is successfully demonstrated with those facilities. The viability of using the gray-scale technology in the photomasks for the fabrication of 3D microstructures is also reported. Moreover, SU-8 microstructures for different applications are shown throughout the paper.
\end{abstract}

Keywords: SU-8; UV photolithography; low-cost microfabrication; without cleanroom facility; microfluidic; MEMS

\section{Introduction}

The field of Microtechnology and Micro-Electro-Mechanical Systems (MEMS) has grown rapidly in the last few years [1]. The beginning of microtechnology relies, mainly, in micromachining from 
glass or silicon. Although these technologies offer high precision, their fabrication methods are often complex, time consuming, and sometimes costly, which boosted research into alternative materials [2]. Nowadays, polymers have emerged as preferential material for microstructures fabrication due to their low-cost and easy processing [1-3]. In particular, epoxy-based negative photoresist (SU-8) is used as a structural and mold material. Its photosensitivity allows its patterning by Ultraviolet Rays (UV) photolithography and its transparency to visible light makes it compatible with optical detection systems. Moreover, it is biocompatible and mechanically and chemically stable $[4,5]$.

SU-8 was originally developed by IBM in Yorktown in the late 1980s and was designed for the fabrication of microstructures of high aspect ratios [3]. Currently, it is commercially available from Microchem Corporation (Westborough, MA, USA) [6] and Gersteltec Sàrl (Pully, Switzerland) [7] in various formulations that cover thicknesses from 1 to $600 \mu \mathrm{m}$. Despite that its use for the fabrication of microstructures is specified by a protocol available at the suppliers, in practice, SU-8 is very sensitive to the processing parameters, therefore, those protocols involve optimizations when the environmental and tools' conditions are changed, as well as according to the structural dimensions and the design of the microstructure. In this paper, it is reported processing values that are crucial for achieved SU-8 microstructures with a low-cost process (masks and equipment) and without the use of a cleanroom. The fabrication of 3D SU-8 microstructures, using gray-scale masks, is also addressed. Furthermore, the applications of SU-8 for microfluidic and MEMS are also demonstrated.

\section{SU-8 Processing Using Low-Cost Facilities}

The processing of SU-8 is a challenging task that involves several lithographic methods being the most common the UV radiation, although it can be used X-ray, electron and protons beams [3]. Each step of the process strongly influences the results and must be individually tailored to the desired application. The patterning of the SU-8 by lithography allows microstructures of almost any shape and size; not only planar microstructures, but also multilayer and 3D microstructures [8]. For that, several lithographic techniques can be used, such as, layer-by-layer multi exposure lithography [8,9], inclined/rotated lithography [8,10], holographic lithography, and gray-scale lithography [8,11].

Figure 1 shows the SU-8 processing steps and the respective equipment used that allows the fabrication of microstructures with aspect ratios of more than 20, with low-cost and without cleanroom facility. Details of each step will be discussed in Section 2.1. The remove and hardbake steps are optional steps. The hardbake is used to further harden the SU-8 structure when it is used in a final device. The remove step is used to obtain free SU-8 microstructures, i.e., without the substrate. In this case it is necessary to deposit a layer of Omnicoater after the substrate preparation and before the SU-8 deposition [12]. All the microstructures reported throughout this paper were fabricated using that equipment.

Despite the processing of SU-8 is described in the datasheets of suppliers, experimentally, the equipment and the environmental conditions differ from those presented in the protocols. Therefore, it is necessary to find optimal processing conditions at each microfabrication laboratory, especially when no cleanroom is available. Thus, this work involved the study and optimization of the SU-8 processing using

four types of SU-8: SU-8 2, SU-8 25, SU-8 100, and SU-8 2150, at different room temperatures (ranging from 19 to $27^{\circ} \mathrm{C}$ ) and for different aspect ratio of the microstructures. All experimental details are presented. It is necessary to mention that, throughout the process, the SU-8 should be less exposed to room light. 
Figure 1. Basic steps of the epoxy-based negative photoresist (SU-8) processing with low-cost and without cleanroom facility.

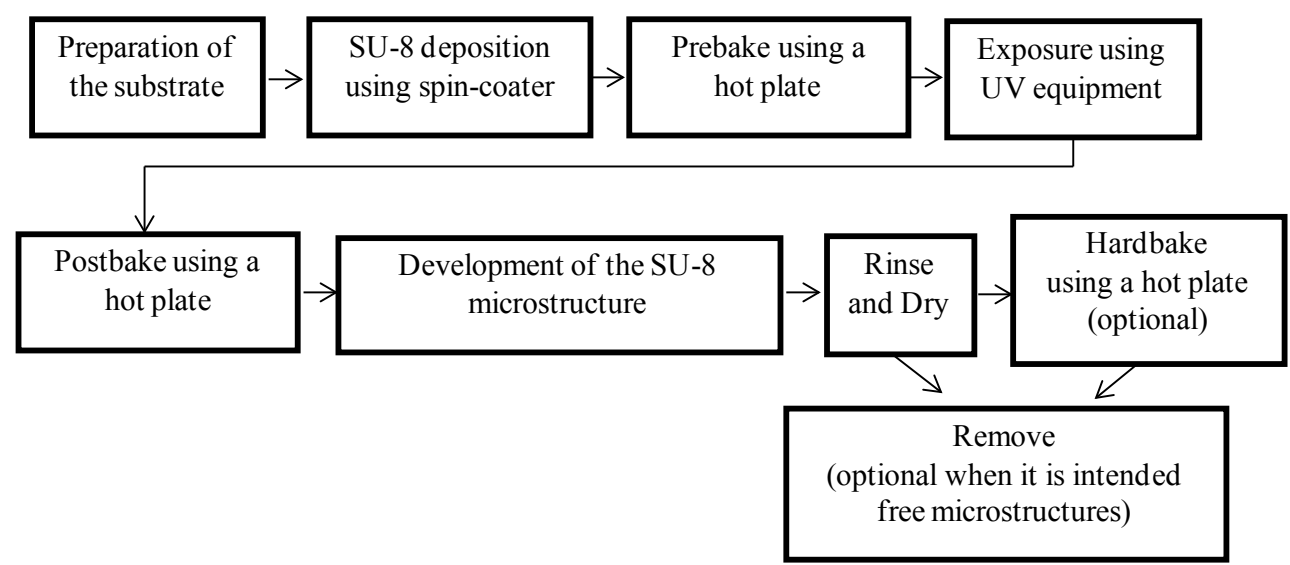

\subsection{Preparation of the Substrate}

The substrates used for the fabrication of SU-8 microstructures are standard microscope glass slides $(76 \times 26 \mathrm{~mm})$ once they are low-cost and exhibit high transparency, which enables their use directly in optical devices. Before use, the glass slides are cleaned with solvents (such as isopropyl alcohol-IPA) followed by drying with a nitrogen stream. In order to dehydrate the surface, the substrates are thermally treated at $200{ }^{\circ} \mathrm{C}$ for 5 min by using a hot plate.

\subsection{SU-8 Deposition}

The SU-8 is deposited using a spin-coater (Polos 200, ATP GmbH, Bienenbüttel, Germany). The influence of the spin-coater rotational speed on the thickness value of the final structure was determined for the four types of SU-8 and for two ranges of room temperature.

The SU-8 2 and SU-8 25 are placed on the center of the glass slides with a Pasteur pipette in an amount of $1 \mathrm{~mL}$. In turn, and due to their high viscosities, the SU-8 100 and SU-8 2150 are weighed and placed on the glass slide. After several experiments, it was found that $1 \mathrm{~g}$ is the appropriate amount of SU-8 in order to obtain a smooth and flat layer across the substrate. The spin-coating deposition is performed in two cycles. The first cycle is set at $500 \mathrm{rpm}$ with a duration time of $7 \mathrm{~s}$ and with an acceleration of $300 \mathrm{rpm} / \mathrm{s}$. The second cycle allows defining the final thickness of the microstructure by adjusting the rotational speed. The thicknesses obtained with rotational speeds of 1000, 2000, and $3000 \mathrm{rpm}$ at acceleration of $300 \mathrm{rpm} / \mathrm{s}$ and during a time of $30 \mathrm{~s}$ are given in Figure 2, for two ranges of room temperature $\left(19-23^{\circ} \mathrm{C}\right.$ and $\left.24-27^{\circ} \mathrm{C}\right)$. It was considered two temperatures ranges once within them the thickness variation is insignificant. The thickness of the fabricated microstructures was characterized using a Veeco Dektak 150 Surface profilometer (Plainview, NY, USA).

The thicknesses obtained with SU-8 2 and SU-8 25 are not influenced by the room temperature, since these types of SU-8 features a very low viscosity. Regarding the SU-8 2150, a thickness of $550 \mu \mathrm{m}$ is obtained with a rotational speed of $1000 \mathrm{rpm}$ and at an ambient temperature between 19 and $23{ }^{\circ} \mathrm{C}$. At higher temperatures $\left(24-27^{\circ} \mathrm{C}\right)$ and for the same rotational speed, microstructures with $370 \mu \mathrm{m}$ thick are obtained. The decrease of the thickness with the temperatures for both SU-8 2150 and SU-8 100 is explained by the decrease of viscosity of the SU-8 with temperature. 
Despite that the SU-8 supplier protocol indicates that each type of SU-8 comprises a specific range of thickness [6], with different environmental and laboratory conditions it is possible to obtain a wider range of thickness than the one referred by the supplier (Table 1).

Figure 2. Thicknesses $v$ s. rotational speeds for (a) SU-8 2 and SU-8 25; (b) SU-8 100 and SU-8 2150, for two ranges of room temperatures.

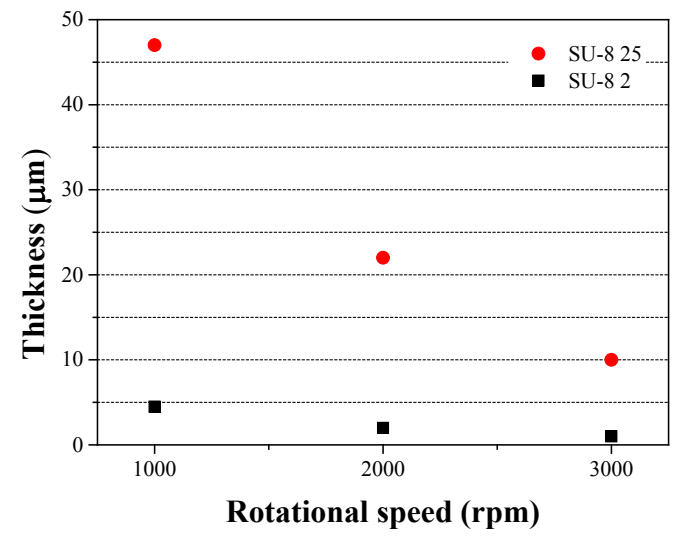

(a)

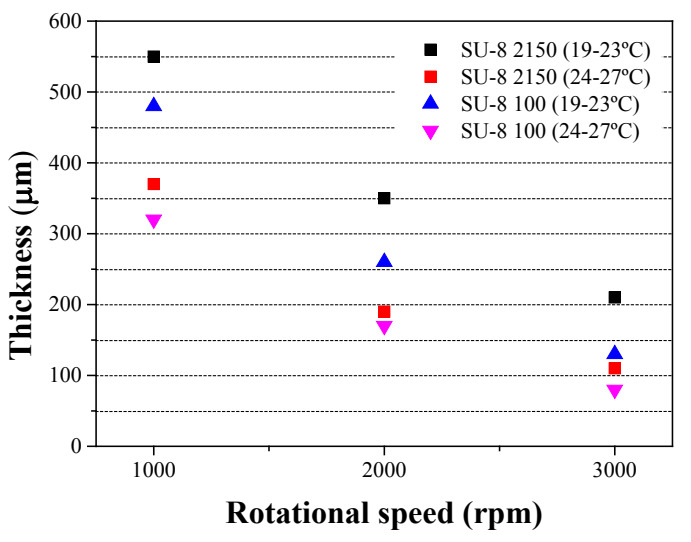

(b)

Table 1. Thicknesses ranges given by supplier and obtained for various types of SU-8.

\begin{tabular}{ccc}
\hline SU-8 type & Thickness range given by supplier $(\boldsymbol{\mu m})[\mathbf{6}]$ & Thickness obtained $(\boldsymbol{\mu m})$ \\
\hline SU-8 2 & $1.5-5$ & $1-5$ \\
SU-8 25 & $15-40$ & $10-47$ \\
SU-8 100 & $100-250$ & $80-480$ \\
SU-8 2150 & $180-550$ & $110-580$ \\
\hline
\end{tabular}

\subsection{Prebake}

The prebake step, responsible for the evaporation of the SU-8 solvent, is performed using a ramping hot plate (Präzitherm type PZ28-2, $1100 \mathrm{~W}$, Harry Gestigkeit GmbH, Düsseldorf, Deutschland). This step involves two cycles. In the first one prebake is done at $65^{\circ} \mathrm{C}$ in order to create a slow evaporation of the solvent resulting in a better coating and a better adhesion to the substrate. The second cycle consists in heating the substrate with the SU-8 layer up to $95{ }^{\circ} \mathrm{C}$, in order to densify the SU-8. The temperature is gradually applied at a rate of $2.5^{\circ} \mathrm{C} / \mathrm{min}$ to decrease the tension induced into the film, to ensure the total evaporation of the solvent and to avoid the occurrence of SU-8 shrinkage after the exposure and cracking formation. Indeed, a quick heating can lead to the incomplete evaporation of the solvent due to the formation of a film on the surface of the SU-8 layer, resulting on tension and shrinkage after UV exposure [3].

Short prebake times cause, as well, an incomplete evaporation of the SU-8 solvent, making small microstructures to move after development, due to the existence of non-polymerized SU-8 at the bottom. The prebake time of each cycle depends on the thickness and type of the SU-8 deposited.

Table 2 shows the optimal times of prebake for each type of SU-8 at different room temperatures. After the prebake, the glass slides with SU-8 are cooled to the room temperature (RT) covered with a dark container. Following Table 2 data carefully, no cracking formation appeared. 
Table 2. Optimal times of prebake for several types of SU-8.

\begin{tabular}{|c|c|c|c|}
\hline \multirow{2}{*}{ SU-8 type } & \multirow{2}{*}{ Final thickness $(\mu \mathrm{m})$} & \multicolumn{2}{|c|}{ Prebake time (min) } \\
\hline & & $65^{\circ} \mathrm{C}$ & $95^{\circ} \mathrm{C}$ \\
\hline \multirow{3}{*}{ SU-8 2} & $1 \pm 0.2$ & 1 & 3 \\
\hline & $2 \pm 0.2$ & 1 & 5 \\
\hline & $4.5 \pm 0.2$ & 1 & 8 \\
\hline \multirow{3}{*}{ SU-8 25} & $10 \pm 3$ & 5 & 7 \\
\hline & $22 \pm 3$ & 5 & 10 \\
\hline & $47 \pm 3$ & 5 & 20 \\
\hline \multirow{3}{*}{ SU-8 100 (RT $\left.19-23{ }^{\circ} \mathrm{C}\right)$} & $130 \pm 10$ & 25 & 45 \\
\hline & $260 \pm 15$ & 35 & 80 \\
\hline & $480 \pm 20$ & 50 & 180 \\
\hline \multirow{3}{*}{ SU-8 100 (RT $\left.24-27^{\circ} \mathrm{C}\right)$} & $80 \pm 10$ & 15 & 35 \\
\hline & $170 \pm 15$ & 30 & 60 \\
\hline & $320 \pm 20$ & 40 & 120 \\
\hline \multirow{3}{*}{ SU-8 2150 (RT $\left.19-23{ }^{\circ} \mathrm{C}\right)$} & $210 \pm 15$ & 15 & 75 \\
\hline & $350 \pm 20$ & 20 & 120 \\
\hline & $550 \pm 30$ & 35 & 180 \\
\hline \multirow{3}{*}{ SU-8 $2150\left(\right.$ RT $\left.24-27^{\circ} \mathrm{C}\right)$} & $110 \pm 10$ & 10 & 45 \\
\hline & $190 \pm 15$ & 15 & 75 \\
\hline & $370 \pm 20$ & 20 & 120 \\
\hline
\end{tabular}

The time of the SU-8 100 prebake is longer compared to the time of the SU-8 2150 prebake even if the amount of solvent is smaller. This is due to the fact that the solvent SU-8 2150 (cyclopentanone), evaporates more rapidly than the SU-8 100 solvent (gamma-butyrolactone).

\subsection{UV Exposure}

The UV exposure is responsible for the generation of a photoacid which cause the polymerization of the SU-8 during the postbake step. In fact, the photoacid opens the epoxide rings of the photoresist and acts as a catalyst in cross-linking reaction [3]. Thus, the UV exposure allows transferring the masks pattern to the SU-8. Chromium masks are the most employed because they present an excellent resolution, $<1 \mu \mathrm{m}$ feature sizes. These masks consist in chromium opaque pattern areas deposited in high quality quartz plate with a very high transparency to UV radiation [13]. However, they have high-cost fabrication [14]. Several authors have conducted studies where inexpensive photomasks are used in order to reduce the overall costs of the photomasks fabrication (including facilities) and, consequently, of the photolithographic processes $[15,16]$. Despite portraying the need for low-cost technologies these approaches use specific equipment and relatively complex processes. To overcome this limitation a cheaper solution ( $€ 10$ per A4 sheet) based in photomasks (high photographic transparent sheets, despite lower than the chromium masks) can be used. This method starts with the design of the desired pattern in a Computer Aided Design (CAD) software and their printing using a laser printer with high resolution (Figure 3). For example, a 2400 dpi printer (Avantra 44s, Agfa, Mortsel, Belgium), which is the most commonly used, allows the fabrication of microstructures with minimum dimensions of $10 \mu \mathrm{m}$ (Figure 4). 
Figure 3. (a) Mask design in the CAD software and (b) photo of a photomask.

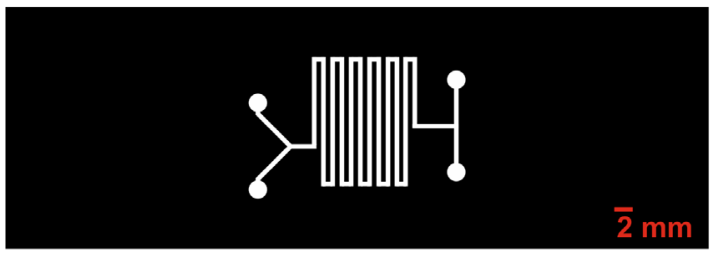

(a)

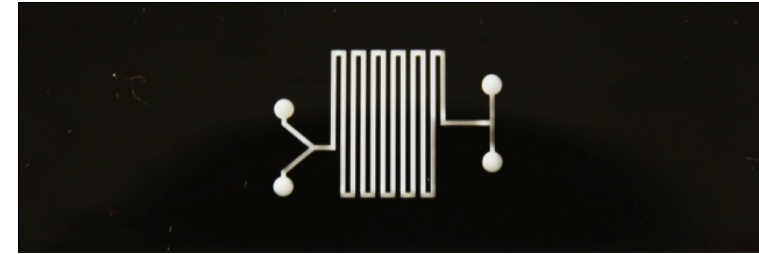

(b)

Figure 4. Photo of a photomask with minimum dimensions of $10 \mu \mathrm{m}$ (the line splitting the squares in the zoom).

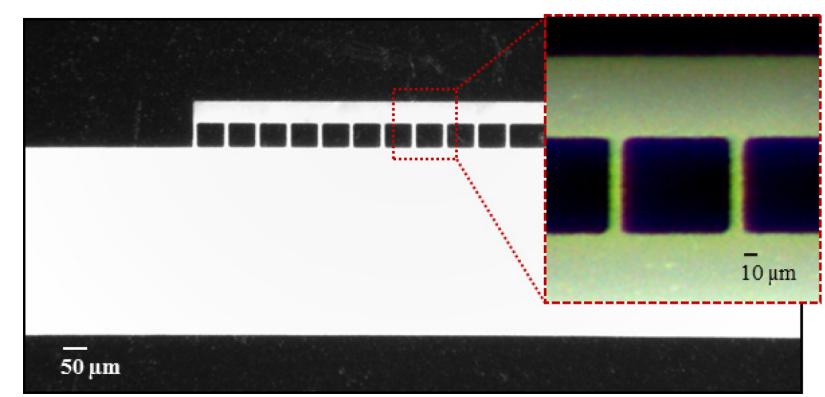

Usually, the equipment used to perform the SU-8 UV exposure is a Mask Aligner. This equipment is expensive, as well as less available, in most laboratories. To overcome this limitation, $10 \mathrm{UV} 20 \mathrm{~W}$ bulbs (Photomeca-type IPP 45, Maxville, France, Figure 5), commonly employed in the Printed Circuit Board (PCB) industry and with an emission range between 347 and 377 nm (FWHM, Full Width Half Maximum $=30 \mathrm{~nm}$ ) is used, which is within the emission range suitable for SU-8 exposure [6]. Moreover, below $331 \mathrm{~nm}$, and after $397 \mathrm{~nm}$, their intensity is zero. Due to their emission range and FWHM values, there is no need for using UV bandpass optical filters, so getting the equipment as used in the PCB industry. Moreover, the obtained structures are well defined, as shown along the paper. That equipment has an air suction system that allows creating a vacuum zone and, therefore, a conformal contact between the mask and the SU-8 without the presence of voids.

The exposure time of SU-8 using that machine is based on Equation (1) [17]. This exposure time served as a reference value and it was optimized according to the results obtained after the development of the final structure in SU-8.

$$
\text { Exposure time }(\mathrm{s})=\frac{\text { Exposure energy }\left(\mathrm{mJ} / \mathrm{cm}^{2}\right)}{\text { Light intensity }\left(\mathrm{mW} / \mathrm{cm}^{2}\right)}
$$

The light intensity of that equipment is $1.2 \mathrm{~mW} / \mathrm{cm}^{2}$. The exposure energy used to determine the exposure time was obtained through the MicroChem datasheets. If the exposure time is inferior to the optimized ones, the microstructures obtained are imperfect. An example is shown in Figure 6. A perfect cross between the vertical and horizontal lines should have been obtained, as shown in the photomask (Figure 6a), contrary to the SU-8 microstructure obtained (Figure 6b).

On the other hand, excessive UV exposure can lead to the hardening of the SU-8 parts that are not exposed since photoacid diffusion can occur. Moreover, it can also cause light diffraction/dispersion effect resulting in severe sidewall distortions [18]. The optimal exposure times using the equipment and tools presented above are shown in Table 3. 
Figure 5. Photography of the UV exposure equipment.

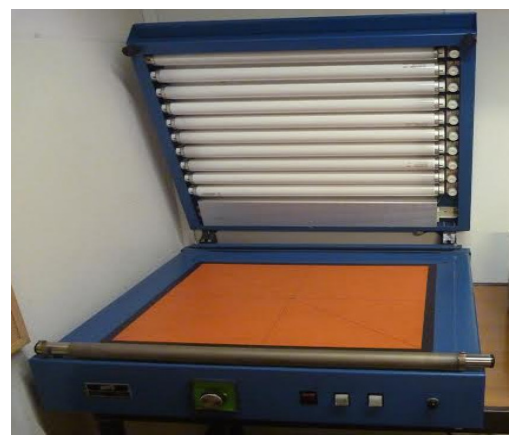

Figure 6. (a) Photomask with the desired pattern; (b) Photography of the SU-8 microstructure obtained with a short exposure time and zoom of the cross lines.

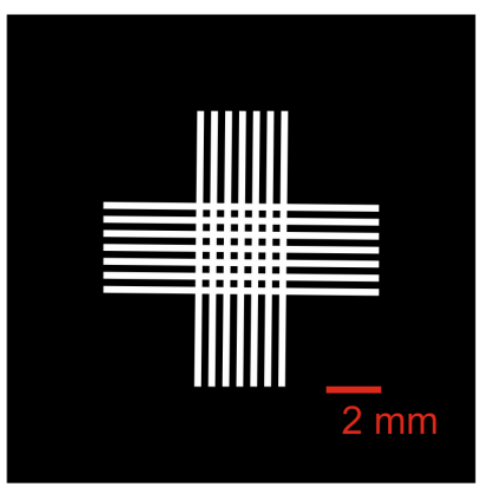

(a)

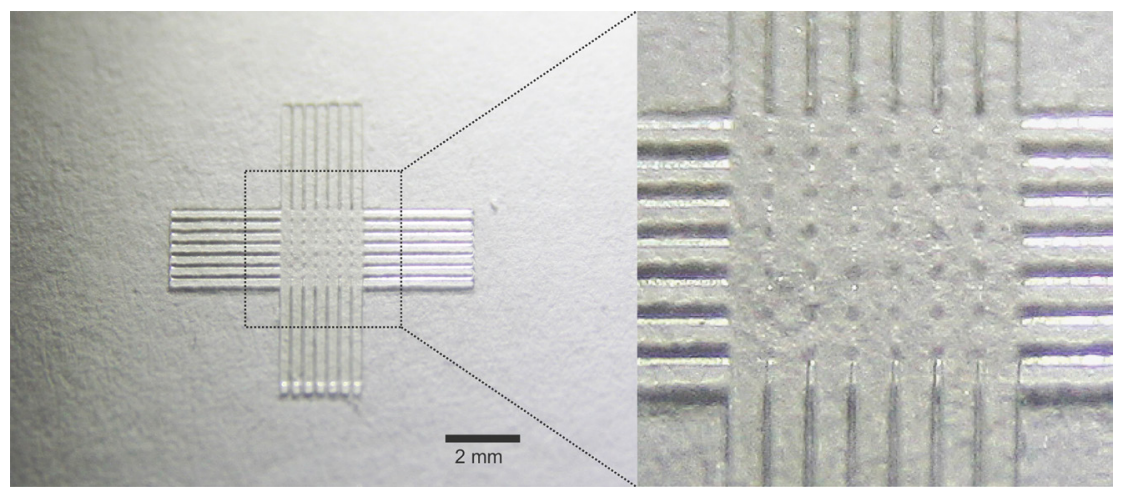

(b)

Table 3. Optimal times of UV exposure for several types of SU-8.

\begin{tabular}{|c|c|c|}
\hline SU-8 type & Final thickness $(\mu \mathrm{m})$ & Exposure time (min) \\
\hline \multirow{3}{*}{ SU-8 2} & $1 \pm 0.2$ & 1 \\
\hline & $2 \pm 0.2$ & 1 \\
\hline & $4.5 \pm 0.2$ & 1.5 \\
\hline \multirow{3}{*}{ SU-8 25} & $10 \pm 3$ & 2 \\
\hline & $22 \pm 3$ & 2 \\
\hline & $47 \pm 3$ & 3 \\
\hline \multirow{3}{*}{ SU-8 100 (RT $\left.19-23^{\circ} \mathrm{C}\right)$} & $130 \pm 10$ & 7 \\
\hline & $260 \pm 15$ & 10 \\
\hline & $480 \pm 20$ & 13 \\
\hline \multirow{3}{*}{ SU-8 $100\left(\right.$ RT $\left.24-27^{\circ} \mathrm{C}\right)$} & $80 \pm 10$ & 5 \\
\hline & $170 \pm 15$ & 8 \\
\hline & $320 \pm 20$ & 11 \\
\hline \multirow{3}{*}{ SU-8 2150 (RT $\left.19-23{ }^{\circ} \mathrm{C}\right)$} & $210 \pm 15$ & 7 \\
\hline & $350 \pm 20$ & 9 \\
\hline & $550 \pm 30$ & 12 \\
\hline \multirow{3}{*}{ SU-8 $2150\left(\right.$ RT $\left.24-27^{\circ} \mathrm{C}\right)$} & $110 \pm 10$ & 4 \\
\hline & $190 \pm 15$ & 6 \\
\hline & $370 \pm 20$ & 10 \\
\hline
\end{tabular}




\subsection{Postbake}

Postbake is responsible for increasing the degree of cross-linking in the SU-8 irradiated areas (due to the photoacid generated in the UV exposure step) making it resistant to the action of solvents in the developing step.

Similarly to the preback step, the temperature is applied gradually to reach two specific temperatures: $65{ }^{\circ} \mathrm{C}$ followed by $95{ }^{\circ} \mathrm{C}$. The optimal postbake times according to the thickness of the SU-8 microstructure are shown in Table 4 . After postbake, the substrate must be cooled slowly to prevent thermal shock.

Table 4. Optimal times of postbake for various types of SU-8.

\begin{tabular}{|c|c|c|c|}
\hline \multirow{2}{*}{ SU-8 type } & \multirow{2}{*}{ Final thickness $(\mu \mathrm{m})$} & \multicolumn{2}{|c|}{ Postbake time (min) } \\
\hline & & $65^{\circ} \mathrm{C}$ & $95^{\circ} \mathrm{C}$ \\
\hline \multirow{3}{*}{ SU-8 2} & $1 \pm 0.2$ & 1 & 2 \\
\hline & $2 \pm 0.2$ & 1 & 2 \\
\hline & $4.5 \pm 0.2$ & 1 & 3 \\
\hline \multirow{3}{*}{ SU-8 25} & $10 \pm 3$ & 2 & 4 \\
\hline & $22 \pm 3$ & 2 & 5 \\
\hline & $47 \pm 3$ & 3 & 6 \\
\hline \multirow{3}{*}{ SU-8 100 (RT $\left.19-23^{\circ} \mathrm{C}\right)$} & $130 \pm 10$ & 5 & 10 \\
\hline & $260 \pm 15$ & 10 & 15 \\
\hline & $480 \pm 20$ & 20 & 25 \\
\hline \multirow{3}{*}{ SU-8 $100\left(\right.$ RT $\left.24-27^{\circ} \mathrm{C}\right)$} & $80 \pm 10$ & 5 & 10 \\
\hline & $170 \pm 15$ & 10 & 12 \\
\hline & $320 \pm 20$ & 15 & 20 \\
\hline \multirow{3}{*}{ SU-8 $2150\left(\right.$ RT $\left.19-23{ }^{\circ} \mathrm{C}\right)$} & $210 \pm 15$ & 5 & 15 \\
\hline & $350 \pm 20$ & 10 & 20 \\
\hline & $550 \pm 30$ & 15 & 30 \\
\hline \multirow{3}{*}{ SU-8 $2150\left(\right.$ RT $\left.24-27^{\circ} \mathrm{C}\right)$} & $110 \pm 10$ & 5 & 10 \\
\hline & $190 \pm 15$ & 5 & 15 \\
\hline & $370 \pm 20$ & 10 & 25 \\
\hline
\end{tabular}

\subsection{Development}

To complete the process and obtained the final SU-8 microstructure, it is necessary to remove the non-polymerized SU-8. For that, the microstructures are immersed in a container with SU-8 Developer from Microchem. Magnetic agitation was used to enhance the removal efficiency of the non-exposed SU-8.

The development times are crucial in order to obtain well-defined SU-8 microstructures. While short development times imply that the non-polymerized SU-8 remains in the structure, longer development times can damage the structure and cause its release from the substrate. The development times are presented in Table 5. However, this step requires a final confirmation of the development using IPA, followed by a nitrogen beam. Evidence of a white color, after this confirmation step, means that the structure has not been fully developed and, therefore, the microstructure must be immersed 
again into the developer solution. This wash process is repeated until there is no white color. The IPA allows the confirmation of the development degree and also confirms the end of the development. A final confirmation step can be performed using optical microscopy.

Table 5. Optimal times for the development step for various types of SU-8.

\begin{tabular}{ccc}
\hline SU-8 type & Final thickness $(\boldsymbol{\mu m})$ & Development time (min) \\
\hline & $1 \pm 0.2$ & 1 \\
SU-8 2 & $2 \pm 0.2$ & 1 \\
& $4.5 \pm 0.2$ & 1 \\
\hline & $10 \pm 3$ & 2 \\
SU-8 25 & $22 \pm 3$ & 3 \\
& $47 \pm 3$ & 5 \\
\hline & $130 \pm 10$ & 10 \\
SU-8 $100\left(\mathrm{RT} 19-23^{\circ} \mathrm{C}\right)$ & $260 \pm 15$ & 20 \\
& $480 \pm 20$ & 35 \\
\hline & $80 \pm 10$ & 7 \\
SU-8 $100\left(\mathrm{RT} 24-27{ }^{\circ} \mathrm{C}\right)$ & $170 \pm 15$ & 12 \\
& $320 \pm 20$ & 25 \\
\hline & $210 \pm 15$ & 20 \\
SU-8 2150 (RT $\left.19-23{ }^{\circ} \mathrm{C}\right)$ & $350 \pm 20$ & 25 \\
& $550 \pm 30$ & 40 \\
\hline & $110 \pm 10$ & 10 \\
SU-8 2150 (RT 24-27 $\left.{ }^{\circ} \mathrm{C}\right)$ & $190 \pm 15$ & 30 \\
\hline
\end{tabular}

For applications where the SU-8 structure is part of the final device, a hardbake step should be done. For that, the SU- 8 microstructure must be heated at a temperature in the range of $150{ }^{\circ} \mathrm{C}$ to $200{ }^{\circ} \mathrm{C}$ and for a time between 5 to $30 \mathrm{~min}$ using a hot plate. The times of hardbake depends on the thickness of SU-8 microstructure.

After all these processing steps, the final SU-8 microstructure is obtained. Figure 7 presents photographs and scanning electron microscope (SEM) images of some SU-8 microstructures fabricated following all the process and the optimizations described. The SEM images were obtained using a Scanning Electron Microscope (Model S360, Cambridge instruments, Cambridge, UK).

The process was optimized for various thicknesses and various types of SU-8, as well as for several structures. The results show high repeatability and reproducibility for the same environmental conditions and the same type of SU-8. Similar microstructures were processed on different days and proved this fact. 
Figure 7. (a) Photography of SU-8 microstructures in glass substrate with lines of $25 \mu \mathrm{m}$ width and $110 \mu \mathrm{m}$ thickness. Scanning electron microscope (SEM) images of SU-8 microstructures with (b) crossed vertical and horizontal lines with a width of $200 \mu \mathrm{m}$ and a thickness of $20 \mu \mathrm{m}$, (c) micropillar arrays with a diameter of $400 \mu \mathrm{m}$ and a thickness of $140 \mu \mathrm{m}$, and (d) microwells with a diameter of $10 \mu \mathrm{m}$ and a depth of $4.7 \mu \mathrm{m}$.

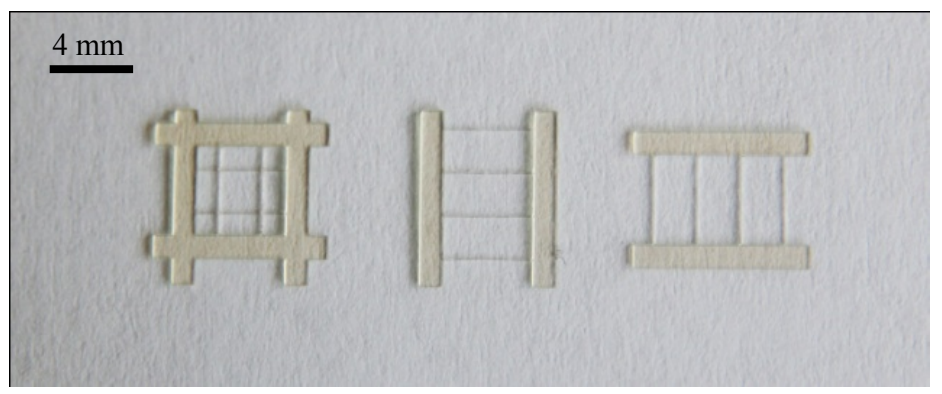

(a)

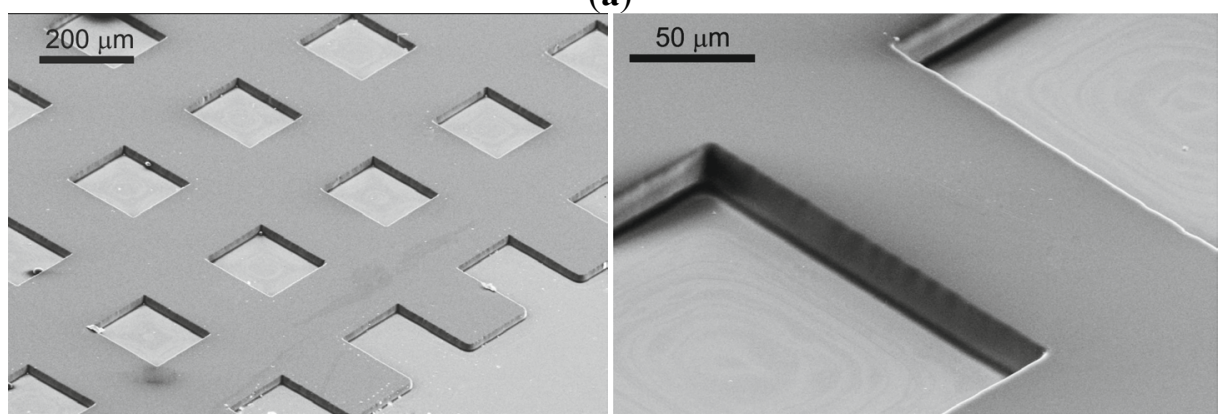

(b)

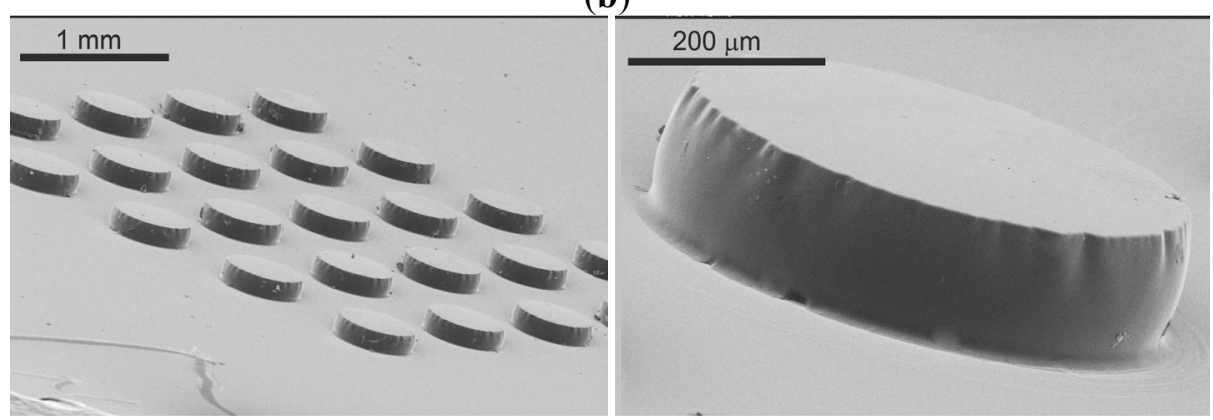

(c)

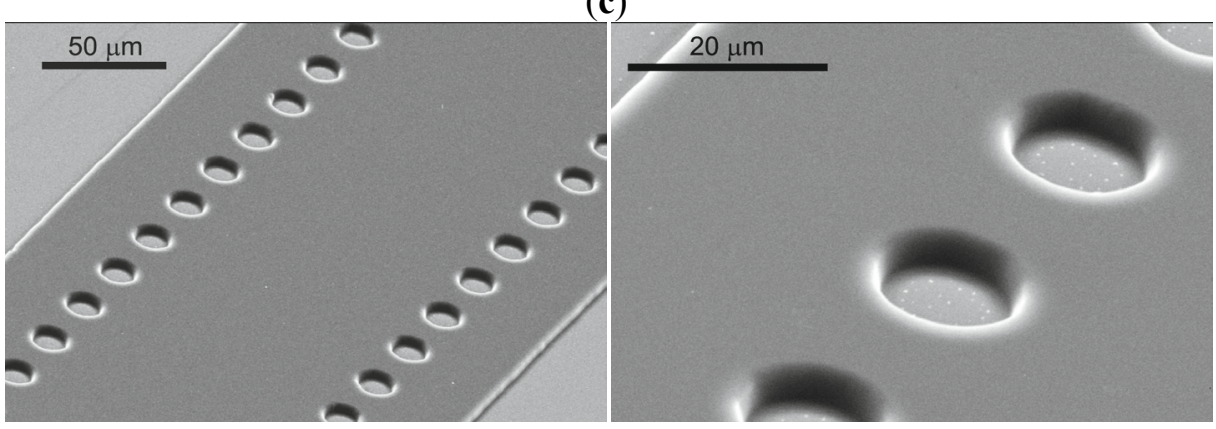

(d)

\subsection{Free Microstructures in SU-8}

For applications requiring the removal of the SU-8 structure from the substrate, a thin layer of Microchem Omnicoat is deposited by spin-coating after cleaning the substrate and prior to the deposition step of the SU-8. For that, $1 \mathrm{~mL}$ of Omnicoat is placed on the glass slide and a rotational 
speed of $500 \mathrm{rpm}$ at an acceleration of $300 \mathrm{rpm} / \mathrm{s}$ during $40 \mathrm{~s}$ is applied. Then, the glass slide is placed on the hot plate at $200{ }^{\circ} \mathrm{C}$ for $5 \mathrm{~min}$. This deposition is repeated 4 times to obtain a thicker layer of Omnicoat in order to facilitate the later removal of the structure. At the end of the fourth layer the glass slide is subjected at a temperature of $200{ }^{\circ} \mathrm{C}$ for $30 \mathrm{~min}$. The subsequent steps are the same as the normal processing of SU-8 (Section 2.2 to 2.6), adding only a final step to remove the Omnicoat after the development step. This removal involves that the structure be immersed in the RemoverPG Microchem until the structure be detached from the glass substrate. Some examples of SU-8 free microstructures fabricated with this process are shown in Figure 8.

Figure 8. Free microstructures in SU-8.
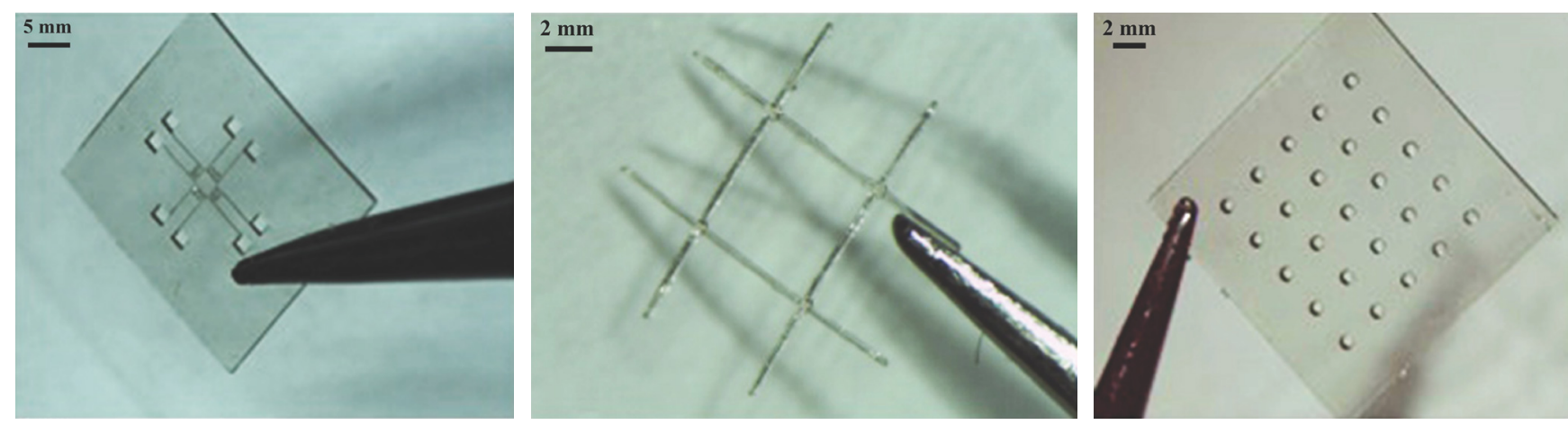

\subsection{Gray-Scale Lithography}

Several methods have been developed to generate 3D microstructures using a coating single layer of SU-8 and with a single UV exposure step. One of these methods is called gray-scale lithography [8]. The gray-scale lithography is typically implemented using gray shades mask allowing the modulation of the exposure dose that reaches the photoresist. With this modulation, different penetration depths of the radiation are obtained resulting in a $3 \mathrm{D}$ structure after development [8,11]. This type of photolithography uses similar steps of the traditional photolithography differing only in the mask used in the UV exposure step. They are gray-scale masks that are fabricated using the dots per inch (dpi) variation across the mask [11]. The gray levels are obtained from pseudo gray-scale, which are generated by dots with different sizes and densities. When the photomask presents low density and small dots (greater transparency), higher exposure doses penetrate the SU-8, which results in a greater final thickness. This density and size of dots is dependent on the resolution of commercial printing equipment. Images of a gray-scale photomask are presented in Figure 9.

Two examples of 3D SU-8 microstructures fabricated by gray-scale lithography are shown in Figure 10a,b, which correspond to the photomasks shown in Figure 9a,b, respectively. To perform the lens-shaped structures of Figure 10b, the mask has few points in the central area, increasing towards the periphery (red square of Figure 9b), which led in higher thickness of the SU-8 in the central area. To demonstrate the curvature degree of the fabricated microstructures a Veeco Dektak 150 Surface profilometer that allows profiles studying was used and the profile is presented in Figure 11. This profile represents a scan over the total surface of one of the lens showed in Figure 10b, the one that has the red dashed arrow, despite Figure 10b show only half lens for better visualization of the curvature. 
Figure 9. Photos of two photomasks with several gray levels.

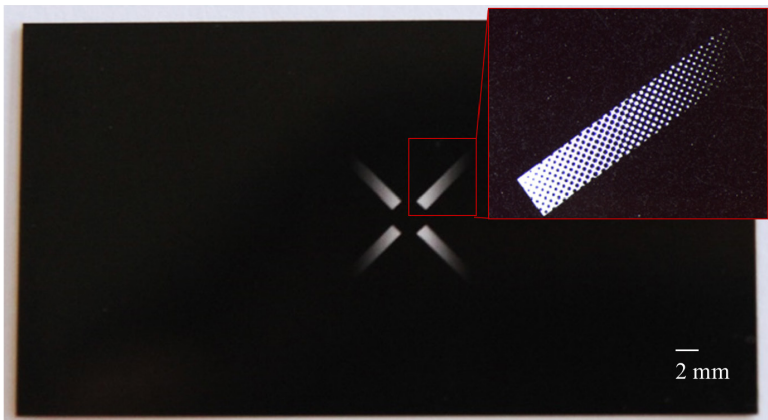

(a)

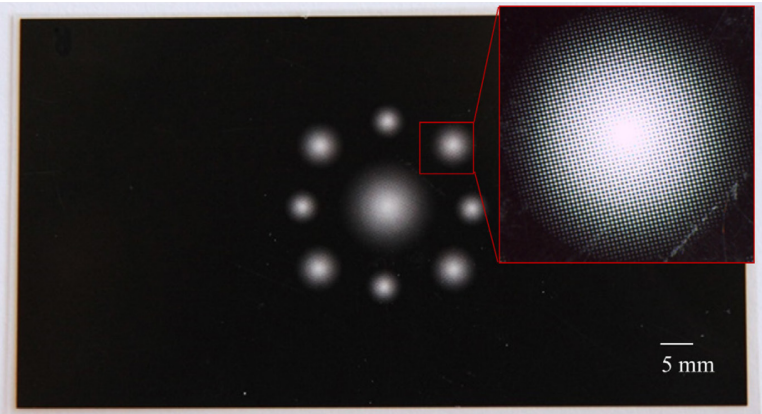

(b)

Figure 10. (a) Inclined and (b) curved SU-8 structure obtained with gray-scale lithography. The red dashed arrow in Figure 10b indicates the lens that was used for the profilometer scan and it corresponds to a distance covered of $535 \mu \mathrm{m}$ to $2700 \mu \mathrm{m}$ in the lens surface, $x$-axis of figure.

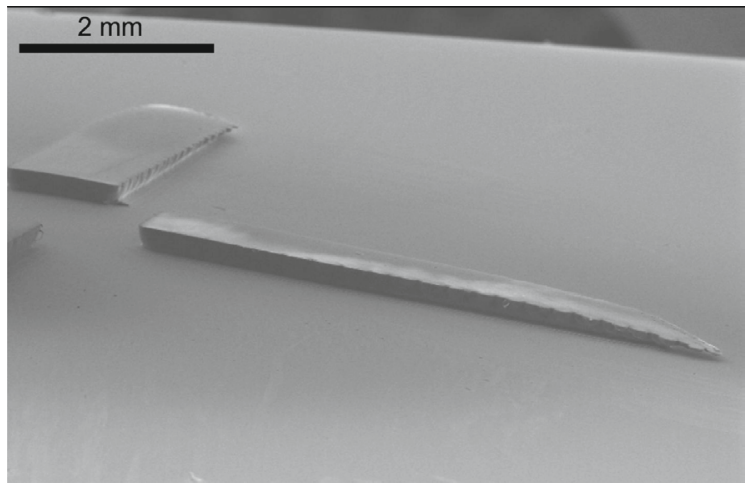

(a)

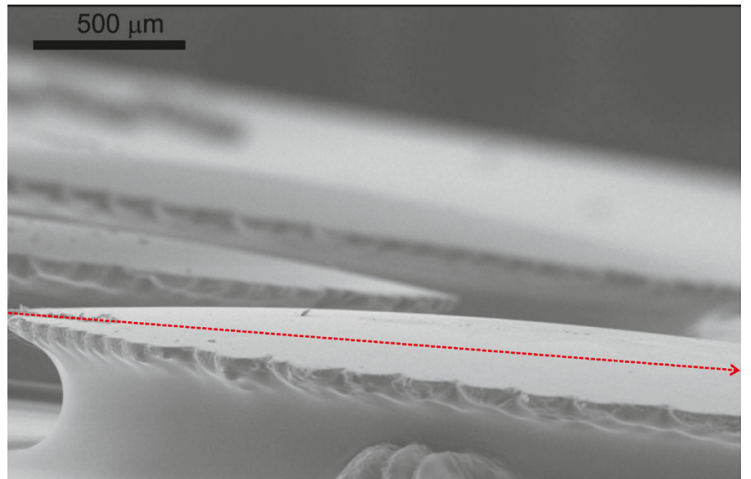

(b)

Figure 11. Profile of the SU-8 microstructure presented in Figure 10b. The red dashed rectangle is the distance covered correspondent to the red dashed arrow of Figure 10b, which means the curvature degree of the lens surface.

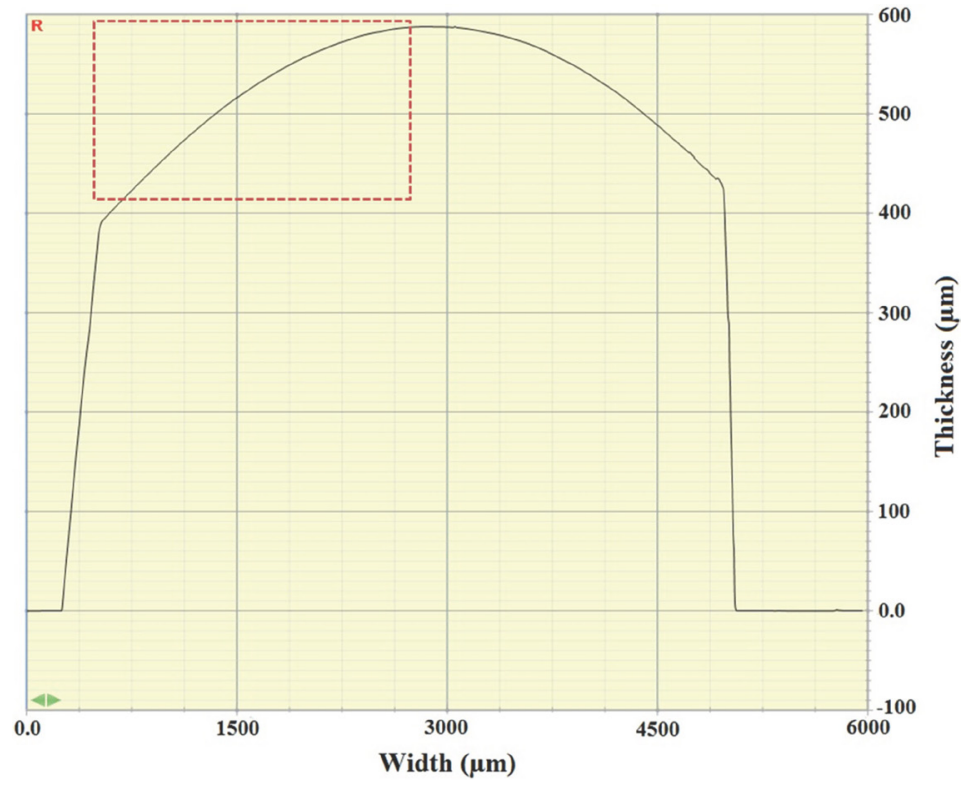




\subsection{Limits of the $S U-8$ Processing}

The SU-8 resolution limits were also tested using the facilities previously described. For this purpose, photomasks with lines of $100,50,25$, and $10 \mu \mathrm{m}$ widths and with different geometries were fabricated. The minimum lateral feature size of $10 \mu \mathrm{m}$ is imposed by the resolution of the commercial print equipment of the photomask. With this study, it was found that SU-8 microstructures with aspect ratios more than 20 are successfully obtained using the processing conditions described above. Photographs and SEM images are shown in Figure 12.

Figure 12. SU-8 microstructures with lines of (a) 300, 200, 100, 50, 40, 30, 20, and $10 \mu \mathrm{m}$ width and of $340 \mu \mathrm{m}$ thick, (b) $300,200,100,50$, and $25 \mu \mathrm{m}$ width and of $23 \mu \mathrm{m}$ thick and (c) $10 \mu \mathrm{m}$ width and $5 \mu \mathrm{m}$ thick.

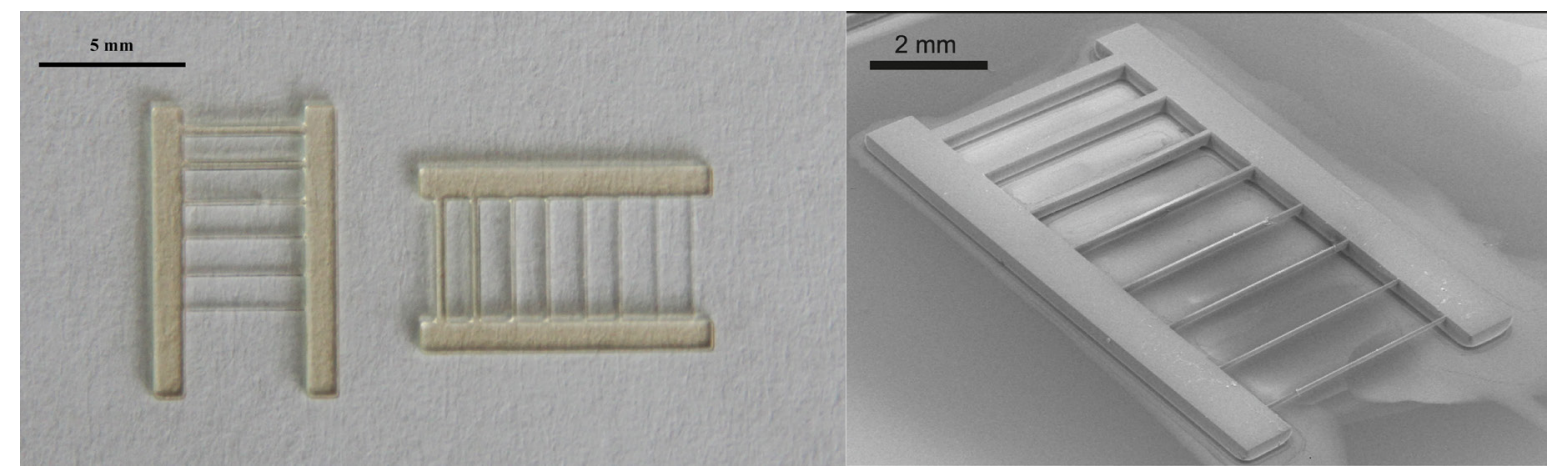

(a)

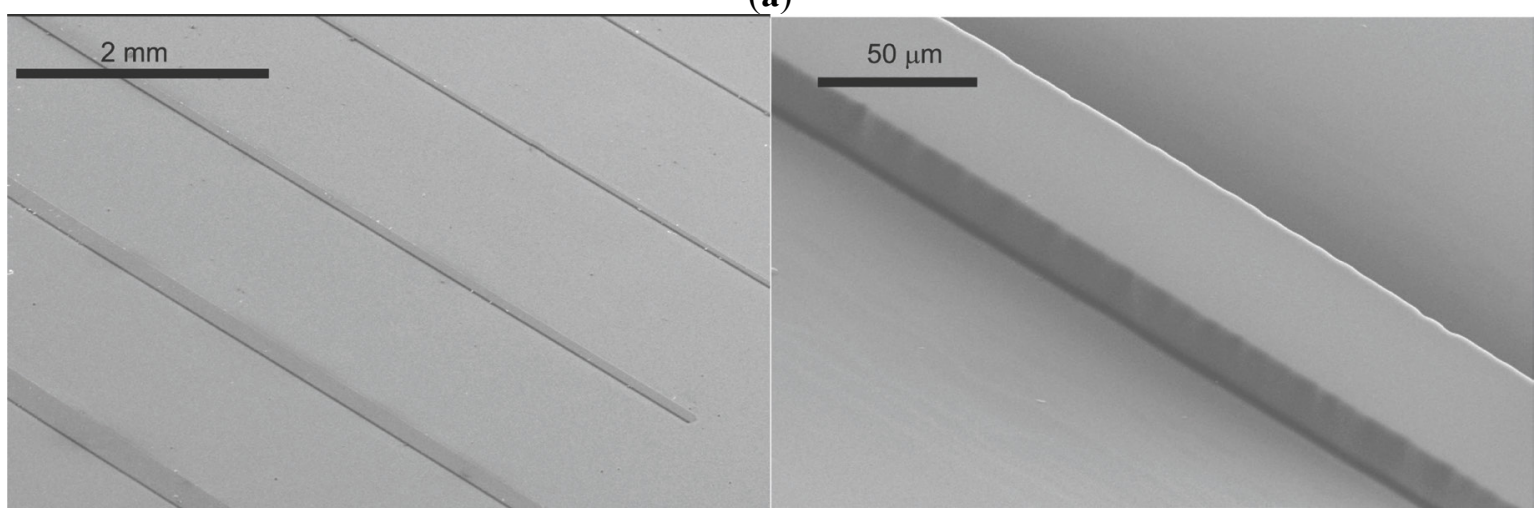

(b)

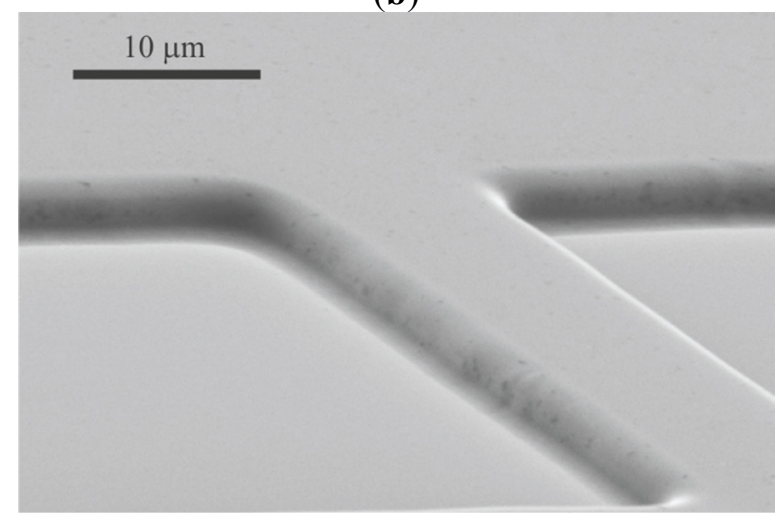

(c) 


\section{Applications of SU-8}

In the last few years, SU-8 has been used in a large range of applications, namely, in the fabrication of microchannels for microfluidic and lab-on-a-chip devices [19-22] and as a structural component in MEMS applications $[4,23,24]$. Some microstructures, fabricated using the process described in Section 2, for the reported applications are briefly shown below.

\subsection{SU-8 as Structural Part}

A SU-8-based diaphragm for piezoresistive pressure sensors is shown in Figure 13a. The structure has a thickness of $110 \mu \mathrm{m}$ and was processed directly in a kapton membrane (50 $\mu \mathrm{m}$ thick). The latter contains patterned metallic thin films, forming the strain gauges pressure sensors. The SU- 8 replaced the kapton as the support material outside the patterned strain gauges due to both its stiffness, which allows supporting all the structure while enabling the free movement of the piezoresistive zone, and to its high adhesion to kapton. This sensor is intended to be included in endoscopic probes for manometry measuring [25].

Other examples of SU-8 structural components include microneedles, as shown in Figure 13b. These microstructures can be used as cantilevers, probes, and micromanipulators, among others.

Figure 13. (a) Piezoresistive pressure sensor with a SU-8 diaphragm and (b) SU-8 microneedles.

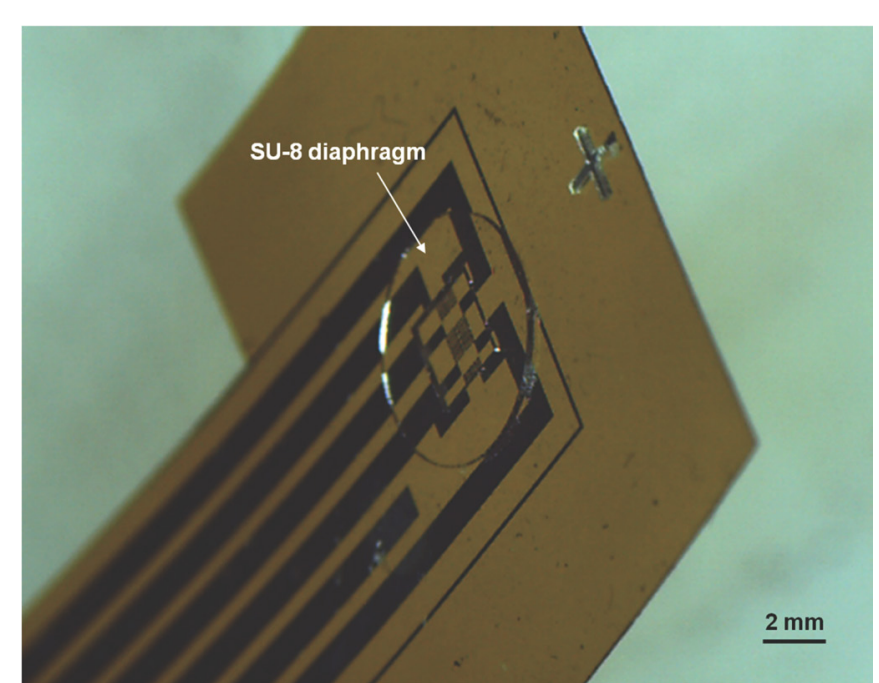

(a)

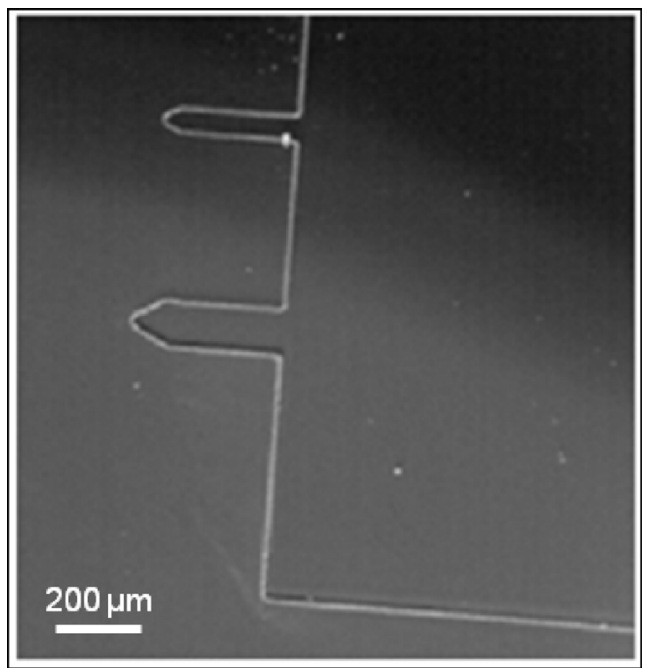

(b)

\subsection{SU-8 as a Mold}

SU-8 has also been used as a mold to fabricate PDMS microfluidic systems by soft lithography techniques since this material has been vastly employed in microfluidic devices due to its low-cost, transparency, and fast fabrication process. Figure 14 shows an example of the SU-8 molds for the fabrication of the PDMS microfluidic devices. The SU-8 molds present the inverse structure of the desired pattern. The PDMS microfluidic devices resultants of these molds are being applied to the purification of biomolecules in aqueous two-phase systems (ATPS) (SU-8 mold of Figure 14a) and for the analysis of analytes in biological fluids (SU-8 mold of Figure 14b). 
Another application is presented in Figure 15. SU-8 molds were fabricated for the production of Polydimethylsiloxane (PDMS) microchannels arrays for integration into a fuel cell. Thus, it is possible to operate with a co-laminar flow, avoiding the use of physical barriers as membranes to separate the anode and the cathode.

Figure 14. SU-8 molds for the fabrication of Polydimethylsiloxane (PDMS) microchannels.

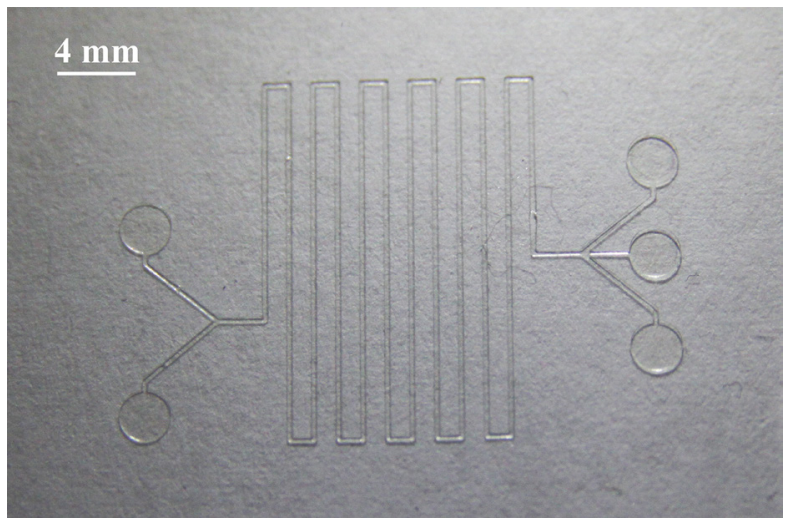

(a)

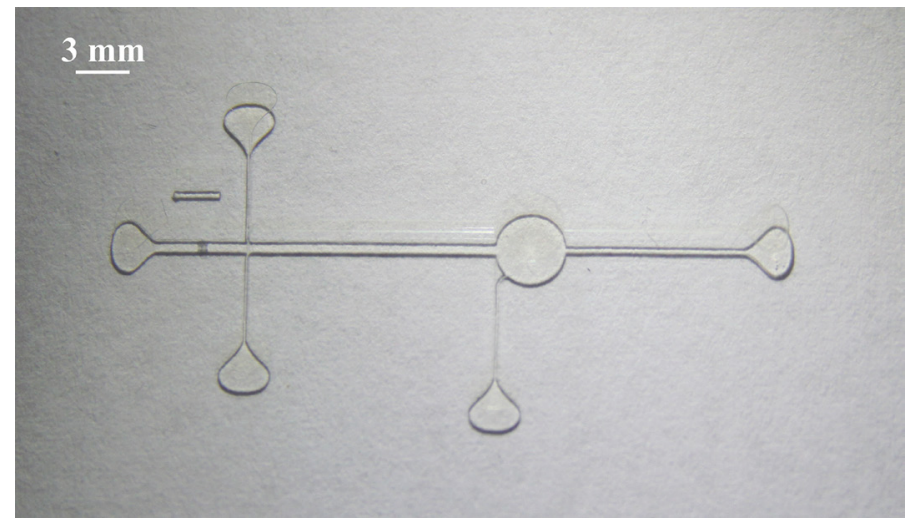

(b)

Figure 15. (a) SU-8 channels mold with a width of $200 \mu \mathrm{m}$ spaced by $50 \mu \mathrm{m}$ and a thickness of $120 \mu \mathrm{m}$. (b) PDMS microstructures resulting from the SU-8 mold.

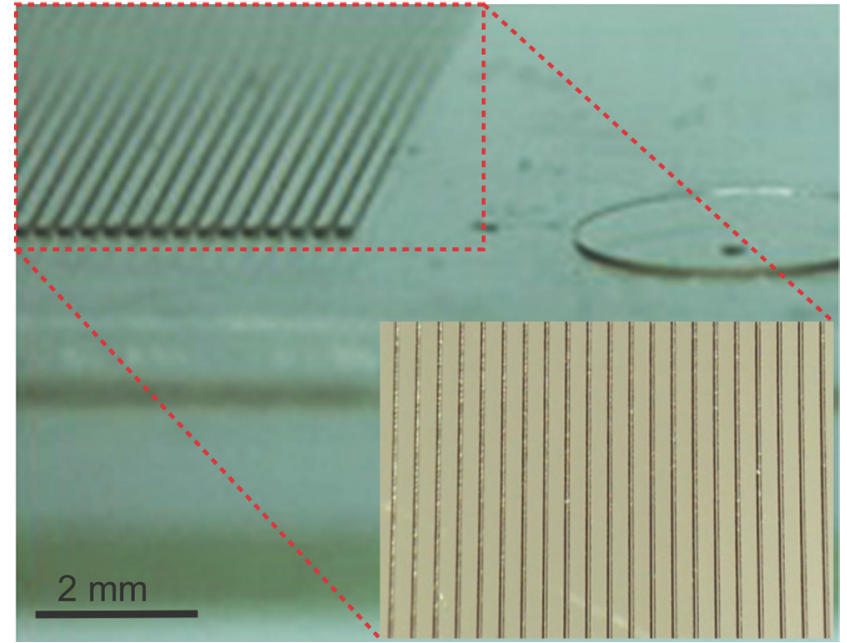

(a)

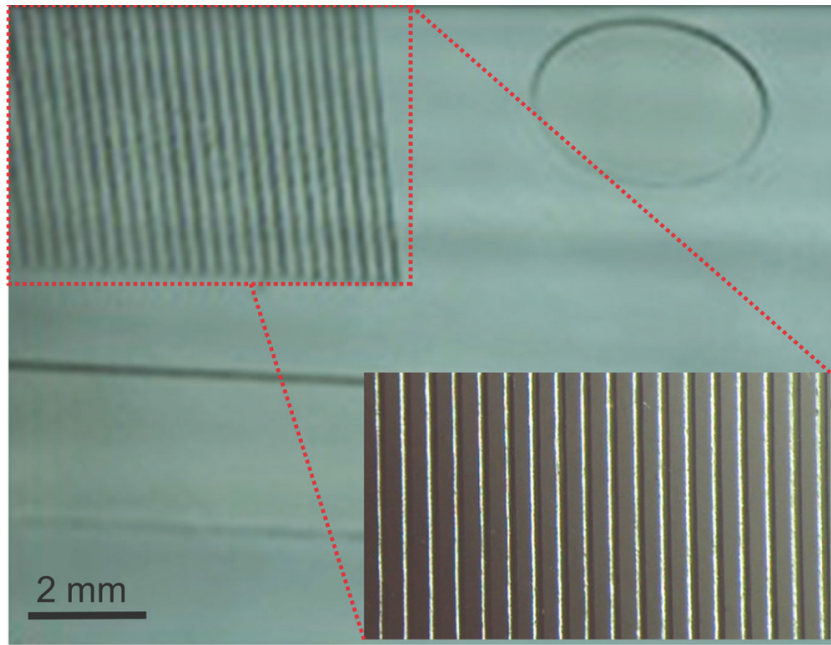

(b)

\subsection{SU-8 Mask for Wet Etching Processes}

Due to the excellent chemical resistance of SU-8, it has also been used as a mask for wet etching of aluminum deposited in Kapton membranes. An example of a SU-8 patterned on the top of a $200 \mathrm{~nm}$ thick aluminium (Al) film allowing subsequent etching of the metal in specific areas using Al etch solution is shown in Figure 16. Figure 16a shows the SU-8 mask over Al before being immersed in the Al etch, while Figure 16b exhibits the Al after the etching step. 
Figure 16. Photographs of (a) SU-8 mask over the Al thin film (b) Al after the etching process.

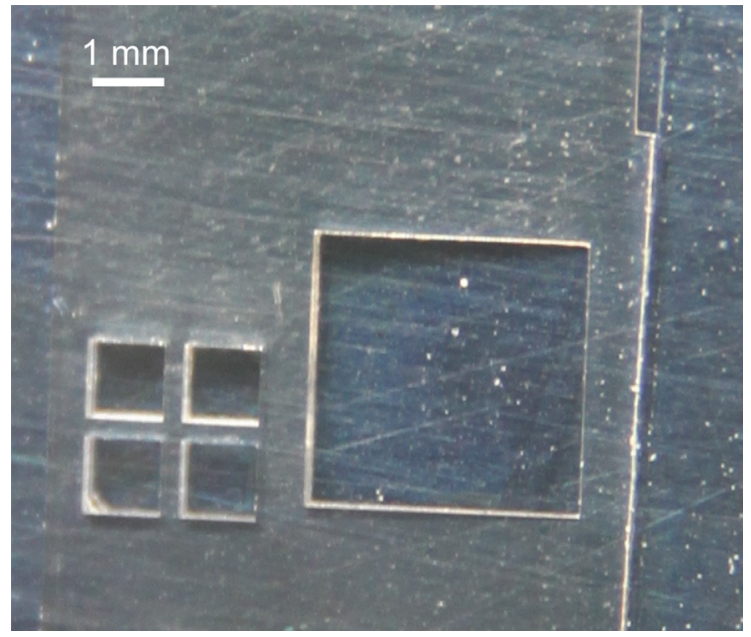

(a)

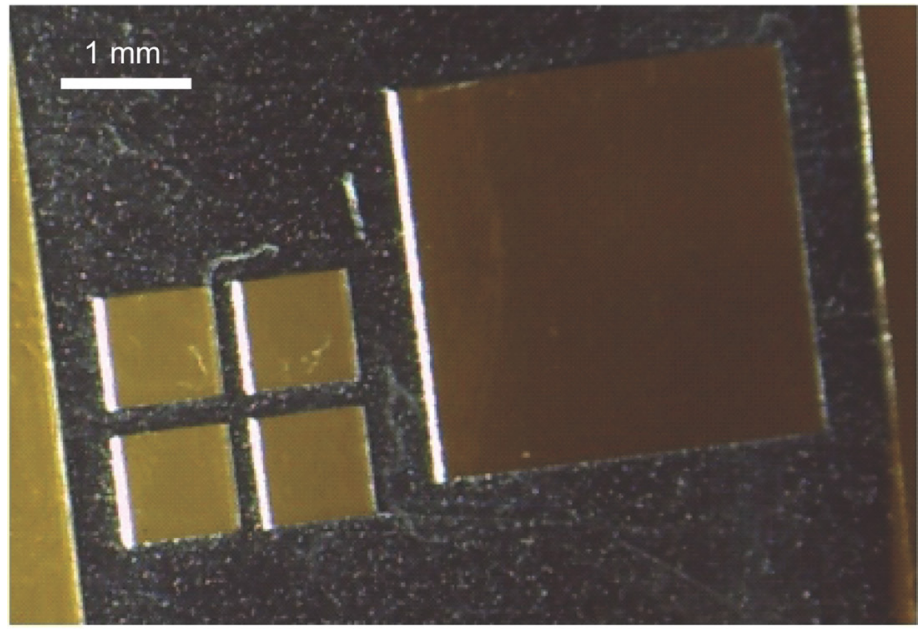

(b)

\section{Conclusions}

This paper presents the fabrication of SU-8 microstructures through a low-cost process and without the need for a cleanroom facility. Simple and low-cost solutions for photolithographic processes are always interesting, especially in microfluidic and MEMS areas, since the main disadvantage of this technique lies in the high cost of processing and equipments. The solutions presented demonstrate the possibility of using alternative photomask printed in transparent photographic sheet, as well as equipment and tools commonly employed in the PCB industry (UV light equipment) for SU-8 microstructures fabrication. The fabrication of well-defined SU-8 microstructures, with a minimum resolution of $10 \mu \mathrm{m}$ and an aspect ratio more than 20, was successfully achieved using this process. The resolution of this process is suitable for most microfluidic applications. Moreover, the gray-scale technology for the fabrication of 3D microstructures has also been demonstrated. All the parameters used in the SU-8 microstructures processing are described, as well as their optimization. Some possible applications of the SU-8 in microfluidic systems and MEMS prove the feasibility of the fabrication process. In microfluidic systems, SU-8 microstructures have been widely used as molds to fabricate PDMS microchannels for several applications including purification and biomolecules analysis, and to integrate fuel cell. In MEMS, the main application consisted in the fabrication of diaphragms for piezoresistive pressure sensors, among others. Therefore, the scientific and academic community that does not have access to clean room facilities could, following some guidelines presented here, implement and optimize the SU-8 processing, according their environmental and tools conditions.

\section{Acknowledgments}

Work supported by FEDER funds through the Eixo I do Programa Operacional Fatores de Competitividade (POFC) QREN, project reference COMPETE: FCOMP-01-0124-FEDER-020241, and by FCT- Fundação para a Ciência e a Tecnologia, project reference PTDC/EBB-EBI/120334/2010. Vânia C. Pinto thanks the FCT for the SFRH/BD/81526/2011 grant. Paulo J. Sousa thanks the FCT for the SFRH/BD/81562/2011 grant. Vanessa F. Cardoso thanks the FCT for the SFRH/BPD/98109/2013 
grant. The authors also acknowledge Luis Silva, from the University of Minho, Centro Algoritmi, for their contribution in the strain gauge applications.

\section{Author Contributions}

Vânia C. Pinto and Vanessa F. Cardoso developed the SU-8 fabrication process and its optimization. The PDMS devices and the strain gauges were performed by Paulo J. Sousa. The applications were set by Graça Minas who supervised the presented work. All authors have analyzed the results and findings. Vânia C. Pinto and Vanessa F. Cardoso have written the main manuscript and all authors reviewed the manuscript.

\section{Conflicts of Interest}

The authors declare no conflict of interest.

\section{References}

1. Hopcroft, M.; Kramer, T.; Kim, G.; Takashima, K.; Higo, Y.; Moore, D.; Brugger, J. Micromechanical testing of SU-8 cantilevers. J. Fatigue Fract. Eng. Mater. Struct. 2005, 28, 735-742.

2. Patel, J.N.; Kaminska, B.; Gray, B.L.; Gates, B.D. PDMS as a sacrificial substrate for SU-8-based biomedical and microfluidic applications. J. Micromech. Microeng. 2008, 18, 095028.

3. Martinez-Duarte, R.; Madou, M.J. SU-8 Photolithography and Its Impact on Microfluidics. In Microfluidics and Nanofluidics Handbook: Fabrication, Implementation, and Applications; Mitra, S.K., Chakraborty, S., Eds.; CRC Press: Boca Raton, FL, USA, 2011; Volume 2, pp. 231-268.

4. Dey, P.K.; Pramanick, B.; RaviShankar, A.; Ganguly, P.; Das, S. Microstructuring of SU-8 resist for MEMS and bio-applications. Int. J. Smart Sens. Intell. Syst. 2010, 3, 118-129.

5. Parida, O.P.; Bhat, N. Characterization of optical properties of SU-8 and fabrication of optical components. In Proceedings of the International Conference on Optics and Photonics, Chandigarh, India, 30 October-1 November 2009.

6. MicroChem. Available online: http://www.microchem.com/ (accessed on 29 May 2014).

7. Gersteltec Engineering Solutions. Available online: http://www.gersteltec.ch/ (accessed on 29 May 2014).

8. Campo, A.D.; Greiner, C. SU-8: A photoresist for high-aspect-ratio and 3D submicron lithography. J. Micromech. Microeng. 2007, 17, R81-R95.

9. Lee, J.A.; Lee, S.W.; Lee, K.C.; Park, S.; Lee, S.S. Fabrication and characterization of freestanding 3D carbon microstructures using multi-exposures and resist pyrolysis. J. Micromech. Microeng. 2008, 18, 035012.

10. Han, M.; Lee, W.; Lee, S.K.; Lee, S.S. 3D microfabrication with inclined/rotated UV lithography. Sens. Actuators A Phys. 2004, 111, 14-20.

11. Rammohan, A.; Dwivedi, P.K.; Martinez-Duarte, R.; Katepalli, H.; Madou, M.J.; Sharma, A. One-step maskless grayscale lithography for the fabrication of 3-dimensional structures in SU-8. Sens. Actuators B Chem. 2011, 153, 125-134. 
12. Lim, Y.C.; Kouzani, A.Z.; Kaynak, A.; Dai, X.J.; Littlefair, G.; Duan, W. A protocol for improving fabrication yield of thin SU-8 microcantilevers for use in an aptasensor. Microsyst. Technol. 2013, doi:10.1007/s00542-013-2019-1.

13. Gale, B.K.; Eddings, M.A.; Sundberg, S.O.; Hatch, A.; Kim, J.; Ho, T. Fabrication and packaging: Low-cost MEMS technologies. In Comprehensive Microsystems, 1st ed.; Gianchandani, Y.B., Tabata, O., Zappe, H.P., Eds.; Elsevier: Amsterdam, The Netherlands, 2008; pp. 1-38.

14. Lau, K.H.; Giridhar, A.; Harikrishnan, S.; Satyanarayana, N.; Sinha, S.K. Releasing high aspect ratio SU-8 microstructures using AZ photoresist as a sacrificial layer on metallized Si substrates. Microsyst. Technol. 2013, 19, 1863-1871.

15. Cabriales, L.; Hautefeuille, M.; Fernández, G.; Velázquez, V.; Grether, M.; López-Moreno, E. Rapid fabrication of on-demand high-resolution optical masks with a CD-DVD pickup unit. Appl. Opt. 2014, 53, 1802-1807.

16. Orabona, E.; Caliò, A.; Rendina, I.; Stefano, L.D.; Medugno, M. Photomasks fabrication based on optical reduction for microfluidic applications. Micromachines 2013, 4, 206-214.

17. Wu, H.; Odom, T.W.; Whitesides, G.M. Generation of chrome masks with micrometer-scale features using microlens lithography. Adv. Mater. 2002, 14, 1213-1216.

18. Kang, W.J.; Rabe, E.; Kopetz, S.; Neyer, A. Novel exposure methods based on reflection and refraction effects in the field of SU-8 lithography. J. Micromech. Microeng. 2006, 16, 821-831.

19. Ribeiro, J.C.; Minas, G.; Turmezei, P.; Wolffenbuttel, R.F.; Correia, J.H. A SU-8 fluidic microsystem for biological fluids analysis. Sens. Actuators A Phys. 2005, 123-124, 77-81.

20. Oliveira, H.C.; Rocha, J.G.; Barros, I.; Cabral, J.M.; Minas, G. A SU-8 microfluidic mixer for use in lab-on-a-chip devices for biological fluids analysis. In Proceedings of 9th International Conference on Industrial Technology, Mumbai, India, 15-17 December 2006; pp. 3014-3019.

21. Huang, Y.; Mason, A.J. Lab-on-CMOS integration of microfluidics and electrochemical sensors. Lab Chip. 2013, 13, 3929-3934.

22. Yu, H.; Balogun, O.; Li, B.; Murray, T.W.; Zhang, X. Fabrication of three-dimensional microstructures based on singled-layered SU-8 for lab-on-chip applications. Sens. Actuators A Phys. 2006, 127, 228-234.

23. Zhang, J.; Tan, K.L.; Gong, H.Q. Characterization of the polymerization of SU-8 photoresist and its applications in micro-electro-mechanical systems (MEMS). Polym. Test. 2001, 20, 693-701.

24. Seidemann, V.; Rabe, J.; Feldmann, M.; Büttgenbach, S. SU8-micromechanical structures with in situ fabricated movable parts. Microsyst. Technol. 2002, 8, 348-350.

25. Silva, L.R.; Sousa, P.J.; Goncalves, L.M.; Minas, G. Pressure Sensor for Gastrointestinal Intraluminal Measuring. In Proceedings of 7th International Conference on Biomedical Electronics and Devices, Angers, Loire Valley, France, 3-6 March 2014; pp. 200-206.

(C) 2014 by the authors; licensee MDPI, Basel, Switzerland. This article is an open access article distributed under the terms and conditions of the Creative Commons Attribution license (http://creativecommons.org/licenses/by/3.0/). 